\title{
Evaluation of the Influence of a Pharmacist-led Patient-Centered Medication Therapy Management and Reconciliation Service in Collaboration with Emergency Department Physicians
}

\author{
Arinze Nkemdirim Okere, PharmD, MS, BCPS; Colleen M. Renier, BS; \\ and James J. Tomsche, PharmD, BCPS
}

\begin{abstract}
BACKGROUND: The implementation of the Patient Protection and Affordable Care Act is anticipated to increase the frequency of emergency department (ED) visits. Therefore, there is a critical need to improve the quality of care transitions among ED patients from ED to outpatient services.

OBJECTIVE: To evaluate the effect of systematic implementation of a pharmacist-led patient-centered approach to medication therapy management and reconciliation service (MRS) in the ED on patient utilization of available health care services.
\end{abstract}

METHODS: A single institution prospective randomized cohort study with 90-day postvisit observation randomized patients into 2 groups:

(1) medication therapy management reconciliation service following a patient-centered approach (MRS) or (2) usual care provided by the institution (non-MRS). To align patient enrollment with availability of other primary care services, subjects were enrolled during weekday daytime hours. Data for the 90 days before and after the index ED visit were matched in all analyses. Generalized estimating equations evaluated any primary care (PC), urgent care (UC), and ED visits during the 90 days post-index ED visit, adjusted by age and sex and weighted by survival time. Generalized linear models evaluated the average number of ED visits during that period, adjusted by age and sex and weighted by survival time. Data were analyzed for all adult patients (ADLTS), aged $\geq 18$ years, and the subpopulation taking 1 or more prescribed daily medication at the time of the index ED visit (ADLTS1+) - the patients expected to receive greatest benefit from an MRS program.

RESULTS: ADLTS MRS patients were 1.9 more likely than non-MRS patients to visit their PC providers (mean difference $0.15, P<0.001$ ). Similarly, ADLTS1+ MRS patients were 1.5 times more likely to visit their PC providers (mean difference $0.10, P=0.026$ ). Although ADLT MRS patients were less likely to visit the UC, this was not significant. However, ADLTS1+ MRS patients were significantly less likely than non-MRS patients $(O R=0.5,95 \%$ $\mathrm{Cl}=0.3-0.9$ ) to visit the UC. No significant difference was seen in ED visits.

CONCLUSIONS: The implementation of a patient-centered approach to medication therapy management and reconciliation improved the odds of patients visiting their PC providers, a positive first step in transitioning patients toward an appropriate use of PC services.

J Manag Care Spec Pharm. 2015;21(4):298-306

Copyright @ 2015, Academy of Managed Care Pharmacy. All rights reserved.

\section{What is already known about this subject}

Frequency of emergency department (ED) use is targeted as a benchmark for quality of transition of care.

Thirty-five percent and 57\% of ED visits occur within 7 and 14 days after an acute care encounter, respectively.

A team approach to patient visits in the ED that includes a physician, nurse, and pharmacist reduced the number of follow-up ED visits.

Pharmacist intervention in the ED has been shown to reduce medication discrepancy and medication errors.

\section{What this study adds}

This study revealed that pharmacist intervention in collaboration with ED physicians decreased utilization of urgent care and enhanced utilization of primary care services.

No overall change was observed in the number of ED visits. The effect of the intervention could have been masked by other ongoing quality improvement initiatives.

The study findings may help provide direction for future research on designing integrated interventions that will influence other health care utilizations of postemergency care visits.

T he number of annual emergency department (ED) visits in the United States increased from approximately 90 million in 1997 to 129 million in 2010.1,2 With the implementation of the Patient Protection and Affordable Care Act, which is designed to cover about 30 million people by 2021, a further increase in ED utilization is anticipated. ${ }^{1}$ According to 1 study, it is expected that the number of ED visits will increase 0.2\%-2.2\%. ${ }^{3}$ Because of increasing health care costs and increased frequency of ED visits, improving appropriate use of the ED has become an area of keen interest among policymakers and hospital administrators.

In addition, some policymakers are advocating the frequency of ED use as a benchmark for quality of transition of care. ${ }^{4,5}$ This is reasonable, since several studies have shown that poor transistion of care from inpatient to outpatient settings and medication discrepancy are primary reasons for ED visits. ${ }^{6-10}$ It is estimated that $40 \%$ of patients return to the ED 
within 30 days after hospitalization, with about 35\% and 57\% of ED visits within 7 and 14 days after an acute care encounter, respectively. ${ }^{11}$ In order to combat the increasing number of ED visits, studies have indicated that improving primary care (PC) access will contribute to the reduction of ED visits. ${ }^{12-16}$ In these examples, Coleman et al. (2001) and Coleman (2003) demonstrated that by improving transistion of care through visits with a care team that included a physician, nurse, and pharmacist the number of ED visits was reduced. ${ }^{4,5}$

The effect of poor transition of care and medication reconciliation has been explored independently. Medication reconciliation has primarily been shown to decrease medication discrepancy. However, studies on medication reconciliation have been limited to collection and documentation of accurate medication histories, and studies on improving transition of care post-ED visit are either expensive or difficult to apply in an ED setting. Little is known about how EDs in an integrated health care system can systematically implement strategies to improve access to PC providers, while improving medication discrepancy during ED visits. In addition, there are no studies demonstrating the role of ED pharmacists in increasing patient utilization of PC services. Hypothetically, systematic interventions that target medication discrepancy and transition of care will impact the rate of ED visits. Following this hypothesis, we conducted a pilot study to demonstrate the effectiveness of proper application of medication reconciliation services following a patient-centered approach. The study showed a reduction in the number of ED visits, improved patient satisfaction, and increased patient-perceived knowledge and confidence in taking medications among patients in the intervention group compared with patients who received usual care. Furthermore, patients who received patient-centered approaches for medication reconciliation were more likely to report that they knew when to contact their PC physicians. ${ }^{17}$

Following the results of the pilot study, we next hypothesized that a pharmacist-led medication therapy management and reconciliation service (MRS) using a patient-centered approach will lead to increased utilization of PC services and a reduction of ED and urgent care (UC) service utilization. The primary purpose of this study was to demonstrate that a pharmacist-led MRS, following a patient-centered approach during an ED encounter, will lead to improvement in the utilization of ED, UC, and PC visits after an ED encounter.

In contrast with the aforementioned pilot study, in which patients were assigned based on convenience sample, this is the first randomized study to evaluate the impact of a pharmacist-led patient-centered provision of medication therapy management and reconciliation during an emergency visit. In this study, a pharmacist-led patient-centered MRS is defined as a face-to-face comprehensive review of all patient home medications prior to admission plus inpatient prescribed and administered medications (during ED encounter and on dis- charge). Given this definition, our approach was developed to proactively optimize all therapies prior to admission for admission encounters and unrelated admission encounters, as well as to discover medication discrepancies, understand patient level of medication compliance and challenges, and provide medication-related education.

\section{Methods}

\section{Design and Setting}

We conducted a randomized cohort study from January 2011 to March 2011, evaluating the role of a pharmacist-led patientcentered MRS, in collaboration with the ED physicians, for patients receiving care in the ED. Patient utilization of health care services was then observed for 90 days following the index ED visit. The study was conducted in a 380-bed community hospital Level II Trauma Center with approximately 33,000 ED visits annually, with data also collected from the affiliated clinic system. This study was approved by the associated Scientific Review Board and Institutional Review Board.

In the usual model of care provided to ED patients (routine care), a triage nurse collects the patient's medication history prior to encounter with a health provider, and discharge counseling is provided by a health provider or by a registered nurse. Inpatient pharmacists are only consulted for specified drug therapy problems or to assist with pharmacokinetics monitoring and evaluation.

Prior to implementation of the MRS, an analysis was made of the utilization patterns of the ED, and the time frame between 8:00 Am and 4:00 pm was identified as the time period with the greatest utilization of these services. A clinical pharmacist was then assigned to the ED during that time frame for the purposes of this study.

\section{Participants}

Initially, study patients were any ED patient, child, or adult with at least 1 weekday ED visit at the appropriate time of day (8:00 Am to 4:00 PM) during the 3-month study period. The index ED visit was the first weekday visit to the ED during the study period. Patients were excluded if they were unconscious at time of admission or cognitively impaired. Additional exclusions included patients with serious mental health conditions, as identified by International Classification of Diseases, Ninth Revision, Clinical Modification (ICD-9-CM) classification and patients with additional preplanned visits. Eligible patients were identified by a research assistant located in the ED and were randomly assigned to either the intervention group (MRS) or the usual care group (non-MRS). The medical record numbers of enrolled patients in both groups were documented to facilitate follow-up observation and to prevent re-enrollment of the same patient if multiple ED visits occurred. Current analysis was restricted to the adult patient population, which contains slightly more MRS than non-MRS patients. 


\section{Randomization Process}

Prior to the study period, a computer-generated MRS/NonMRS Assignment List was created by the biostatistician. This list was provided to the research assistant for randomizing patients to the 2 study groups: the MRS (intervention) group and the non-MRS (usual care) group. Randomization was not stratified by age group-child (aged $<18$ years) and adult (aged $>18$ years) - nor was it possible to consider the primary diagnosis or number of daily medications, which were only available at the conclusion of the ED visit. The investigators were not involved in the patient randomization and selection process. Similarly, patients were blinded during the randomization and selection process. The MRS group consisted of ED patients who received medication management and reconciliation following a patient-centered approach, while the non-MRS group consisted of those who received routine ED care only.

\section{Exposure/Intervention}

Patients randomized to the MRS group received MRS from a pharmacist in collaboration with the ED physician. A patientcentered approach to MRS as provided by the pharmacist must follow 5 essential steps:

1. Patient profile review: Pharmacist reviewed patient's medication and medical history profile, the reasons for ED admission (chief complaint), and laboratory results (if already completed).

2. Verification through patient (or caregiver) interview: Pharmacist verified patient's medication adherence pattern. This was accomplished by face-to-face interviews and verification from patient's primary/secondary pharmacies where prescriptions were filled.

3. ED medication therapy management: Discrepancies were documented and reported. These discrepancies included noncompliance and core medications (based on compelling indications) that were not included in patient's therapeutic regimen. Medication adjustment/error based on patient's comorbidities were discussed with the ED physician. Home and prescribed medications were optimized based on current guidelines.

4. Patient education: At the end of the ED encounter, a complete list of medications and education were provided to patient prior to discharge.

5. Communication to PC providers: Important future recommendations and other patient-care and drug-related issues were documented by the pharmacist in the electronic health record. This was either electronically communicated or mailed to the PC provider when possible. To enable communication between the PC provider and the patient after discharge, a copy of the same letter that had been sent to the $\mathrm{PC}$ provider was also given to the patient. For patients without a documented PC provider, similar letters were given to these patients for them to identify and work with a PC pro-

\begin{tabular}{|c|c|c|}
\hline \multicolumn{3}{|l|}{ Screened $(\mathrm{N}=544)$} \\
\hline neligible for inclusion, $\mathrm{n} \%$ & 33 & 6.1 \\
\hline Baseline survey data incomplete or unusable & 17 & 3.1 \\
\hline Randomization error & 4 & 0.7 \\
\hline $\begin{array}{l}\text { Serious mental health issue identified in the past } 90 \\
\text { days }\end{array}$ & 10 & 1.8 \\
\hline $\begin{array}{l}\text { Serious memory/neorologic/cognition issue in the past } \\
90 \text { days }\end{array}$ & 2 & 0.4 \\
\hline Eligible subjects & 511 & 93.9 \\
\hline
\end{tabular}

\begin{tabular}{|c|c|c|}
\hline \multicolumn{3}{|c|}{ Eligible subjects $(\mathrm{N}=511)$} \\
\hline Intervention status, $\mathrm{n} \%$ & & \\
\hline Non-MRS & 233 & 45.6 \\
\hline MRS & 278 & 54.4 \\
\hline
\end{tabular}

Primary enrollment diagnosis: pregnancy/childbirth/puerperium ${ }^{a}$ (ICD-9-CM codes 630-679), n \%

\begin{tabular}{l|l|l}
\hline Non-MRS & 8 & 3.4 \\
\hline MRS & 6 & 2.2 \\
\hline
\end{tabular}

${ }^{a}$ Removed from further analysis because subsequent events are expected.

ICD-9-CM = International Classification of Diseases, Ninth Revision, Clinical

Modification; MRS = medication therapy management and reconciliation service.

vider. All patients were made aware of the information that would be communicated to their PC providers or should be communicated to their PC providers if no PC provider was indicated in their records.

\section{Main Outcome and Measures}

This study was designed to measure the following endpoints at the study tertiary care institution during the 90 days postindex ED visit: (a) any PC visits, (b) any UC visits, (c) any ED visits, and (d) average number of ED visits (rate per 1,000 persons) during the 30-day periods from 1-30, 31-60, and 61-90 days post-index ED visit. Analyses compared data for the period before and after the index ED visit, adjusted for age and sex, with MRS patients compared with non-MRS patients. As previously stated, analysis was restricted to the adult patient population.

\section{Data Collection}

To evaluate the impact of MRS on patients' subsequent ED visits at the study tertiary care institutions, the institution's electronic medical records were queried. Data collected included PC, UC, and ED visits. Clinic electronic medical records provided data on PC and UC visits 90 days before and after the index ED visit. Hospital electronic medical records provided data for ED visits for the 90 days before the index ED visit, as well as 1-30, 31-60, and 61-90 days post-index ED visit. In addition, a baseline survey collected demographic information and categorization of the number of prescribed medications taken daily. 


\section{Evaluation of the Influence of a Pharmacist-led Patient-Centered Medication Therapy Management and Reconciliation Service in Collaboration with Emergency Department Physicians}

\section{TABLE 2 Adult Sample Baseline Characteristics by Intervention Status}

\begin{tabular}{|c|c|c|c|c|c|c|c|c|}
\hline \multirow{3}{*}{$\begin{array}{l}\text { Variable } \\
\text { Sex, female, n (\%) }\end{array}$} & \multicolumn{4}{|c|}{ All Adults } & \multicolumn{4}{|c|}{$\begin{array}{c}\text { All Adults with 1+ } \\
\text { Medications at Index ED Visi }\end{array}$} \\
\hline & \multicolumn{2}{|c|}{$\begin{array}{c}\text { Non-MRS } \\
\mathrm{n}=225\end{array}$} & \multicolumn{2}{|c|}{$\begin{array}{c}\text { MRS } \\
\mathrm{n}=272\end{array}$} & \multicolumn{2}{|c|}{$\begin{array}{c}\text { Non-MRS } \\
\mathrm{n}=146\end{array}$} & \multicolumn{2}{|c|}{$\begin{array}{c}\text { MRS } \\
\mathbf{n}=\mathbf{2 5 9} \\
\end{array}$} \\
\hline & 125 & $(55.6)$ & 173 & $(63.6)$ & 94 & $(64.4)$ & 167 & $(64.5)$ \\
\hline Age, mean (SD)a & 42.2 & $(18.5)$ & 53.6 & $(19.2)$ & 45.6 & $(19.7)$ & 53.8 & $(19.1)$ \\
\hline \multicolumn{9}{|l|}{ Primary diagnosis at index ED visit (ICD-9-CM range), $n(\%)^{b}$} \\
\hline Endocrine/nutritional/metabolic/immune disorders (240-279) & 5 & $(2.2)$ & 7 & $(2.6)$ & 5 & (3.4) & 7 & $(2.7)$ \\
\hline Mental disorders (290-319) & 5 & $(2.2)$ & 6 & $(2.2)$ & 5 & (3.4) & 6 & $(2.3)$ \\
\hline Diseases of nervous system and sense organs (320-389) & 8 & $(3.6)$ & 16 & $(5.9)$ & 5 & $(3.4)$ & 16 & $(6.2)$ \\
\hline Diseases of circulatory system (390-459) & 5 & $(2.2)$ & 17 & $(6.3)$ & 3 & $(2.1)$ & 17 & $(6.6)$ \\
\hline Diseases of respiratory system $(460-519)$ & 20 & $(8.9)$ & 15 & $(5.5)$ & 13 & $(8.9)$ & 14 & $(5.4)$ \\
\hline Diseases of digestive system (520-579) & 30 & $(13.3)$ & 21 & $(7.7)$ & 21 & $(14.4)$ & 20 & $(7.7)$ \\
\hline Diseases of genitourinary system (580-629) & 14 & $(6.2)$ & 9 & $(3.3)$ & 12 & $(8.2)$ & 7 & $(2.7)$ \\
\hline Diseases of the musculoskeletal system and connective tissue (710-739) & 22 & $(9.8)$ & 27 & $(9.9)$ & 15 & $(10.3)$ & 26 & $(10.0)$ \\
\hline Symptoms/signs/ill-defined conditions (780-799) & 61 & $(27.1)$ & 102 & $(37.5)$ & 39 & $(26.7)$ & 99 & $(38.2)$ \\
\hline Injury (800-999) & 46 & $(20.4)$ & 35 & $(12.9)$ & 23 & $(15.8)$ & 32 & $(12.4)$ \\
\hline Otherc & 9 & $(4.0)$ & 17 & $(6.3)$ & 5 & $(3.4)$ & 15 & (5.8) \\
\hline \multicolumn{9}{|l|}{ Number of prescribed medications taken daily, $\mathbf{n}(\%)^{\mathrm{d}}$} \\
\hline None & 77 & $(34.5)$ & 11 & $(4.1)$ & & - & & - \\
\hline $1-2$ & 62 & $(27.8)$ & 89 & $(33.0)$ & 62 & $(42.5)$ & 89 & $(34.4)$ \\
\hline $3-5$ & 44 & $(19.7)$ & 84 & $(31.1)$ & 44 & $(30.1)$ & 84 & $(32.4)$ \\
\hline $5-9$ & & $(10.8)$ & 59 & $(21.9)$ & 24 & $(16.4)$ & 59 & $(22.8)$ \\
\hline $10+$ & 16 & $(7.2)$ & 27 & $(10.0)$ & 16 & $(11.0)$ & 27 & $(10.4)$ \\
\hline Any hospitalization associated with the index ED visit? $\mathrm{n}(\%)^{\mathrm{d}}$ & 38 & $(16.9)$ & 75 & $(27.6)$ & 32 & $(21.9)$ & 71 & $(27.4)$ \\
\hline \multicolumn{9}{|c|}{$\begin{array}{l}\text { aTwo-tailed } t \text {-test } P<0.05 \text { : all adults and all adults with } 1 \text { or more medications at index ED visit. } \\
{ }^{b} C h i \text {-square } P<0.05: \text { all adults and all adults with } 1 \text { or more medications at index ED visit. } \\
{ }^{c A} \text { combination of remaining ICD-9-CM categories with }<2 \% \text { of all adults. } \\
\text { dChi-square } P<0.05: \text { all adults. } \\
\text { ED=emergency department; ICD-9-CM = International Classification of Diseases, Ninth Revision, Clinical Modification; MRS= medication therapy management and } \\
\text { reconciliation service; } S D=\text { standard deviation. }\end{array}$} \\
\hline
\end{tabular}

\section{Statistical Analysis}

All statistical analyses were conducted using IBM SPSS version 21 (SPSS Inc., Chicago, IL). Study analyses were conducted on the full randomized population and on the subpopulation of patients who reported taking 1 or more prescribed medications daily. This subpopulation would be expected to have the most consistent experience with a medication intervention.

Descriptive statistical analysis, Student's t-test, and chisquare were used to assess MRS and non-MRS demographic and health status differences at the time of the index ED visit. A preset significance level was set as $P<0.05$.

Generalized estimation equations compared the 90-day period before the index ED visit to the 90 days post-index ED visit for any PC, UC, or ED visits. These matched binary models were adjusted for patient sex and age (centered at population mean $=48$ ) and weighted for survival. Differences in ED visits, PC visits, and UC visits between MRS and non-MRS were evaluated.

Mixed models analysis compared the 30-day average number of ED visits (rate per 1,000 persons) from the period before the index ED visit to 1-30, 31-60, and 61-90 days post-index ED visit for both study groups. This repeated measures linear model was adjusted for patient sex and age (centered at population mean $=48$ ).

\section{Results}

Of the approximately 2,000 patients who went through the ED within the 3 months of study, 544 adult patients were seen on weekdays between 8:00 Am and 4:00 PM and met the other preliminary study inclusion and exclusion criteria. Of these, 33 were excluded due to incomplete surveys, randomization errors, severe psychiatric disorders, and serious cognition issues (Table 1). Of the remaining 511 patients, 278 patients were randomized to the MRS group, while 233 patients received routine care as provided in the institution's ED (nonMRS group). Fourteen patients with a diagnosis of pregnancy/ childbirth/puerperium (ICD-9-CM 630-679) were removed prior to analysis because of the pre-existing requirement for additional medical encounters over the next 90 days. 


\begin{tabular}{l|c|c|c|c|c|c|c|c|c|c|c|}
\hline \multirow{2}{*}{} & \multicolumn{9}{c|}{$\begin{array}{c}\text { All Adults } \\
\text { Any Visit }\end{array}$} & \multicolumn{5}{c}{ All Adults with 1+ Medications at Index ED Visit } \\
Any Visit
\end{tabular}

${ }^{a}$ Generalized estimating equations (binary repeated measures): adjusted for age and sex and weighted by proportion of survival time.

${ }^{b}$ All Adults: any $P C$ visit $P<0.05$

cAll Adults with 1+ Medications at Index ED Visit: any $P C$ visit $P<0.05$.

dAll Adults with 1+ Medications at Index ED Visit: any UC visit $P<0.05$

eAll Adults: any UC visit $P<0.05$.

fAll Adults: any ED visit $P<0.05$.

gAll Adults with 1+ Medications at Index ED Visit: any ED visit $P<0.05$.

$C I=$ confidence interval; $E D=$ emergency department; $M R S=$ medication therapy management and reconciliation service; $O R=o d d s$ ratio; $P C=$ primary care; $S E=$ standard error; UC = urgent care.

Data were collected for the remaining 497 patients. Table 2 presents the population characteristics of the remaining 497 eligible adult patients, including the full population (ADLT) and the subpopulation of 405 patients who reported 1 or more prescribed medications taken daily at the time of their index ED visits (ADLT1+) - these were the patients expected to receive the most consistent benefit from the MRS. ALDT and ALDTl + MRS patients were significantly older than nonMRS patients $(53.6 \pm 19.2$ vs. $42.2 \pm 18.5$ and $53.8 \pm 9.1$ vs. $45.6 \pm 19.7$, respectively). ADLT non-MRS patients were also more likely to have been taking no prescribed daily medications (34.5\% vs. $4.1 \%$ ), and ADLT MRS patients were significantly more likely to have been hospitalized in association with the index ED visit. Neither of those differences was present in the ADLT1 + subpopulation.

Generalized estimating equations (binary repeated measures) for any PC, UC, and ED visits for the 90 days before and after the index ED visit, adjusted for sex and age (centered at the population mean =48) and weighted for survival, are presented in Table 3.

\section{Primary Care Utilization}

ADLT MRS patients were 1.9 times (95\% confidence interval $[C I]=1.4-2.6)$ more likely to visit their PC providers, compared with ADLT non-MRS patients (mean difference $0.15, P<0.001$ ). Similarly, ADLT1 + MRS patients were 1.5 (95\% CI $=1.04$ 2.1) times more likely to visit their PC providers than the ADLT1 + non-MRS patients (mean difference 0.10, $P=0.026$ ).
ADLT females were more likely than males to see a PC provider (odds ratio $[\mathrm{OR}]=1.6,95 \% \mathrm{CI}=1.2-2.2$ ); no similar difference was found in the ADLTl + subpopulation. Finally, odds of seeing a PC provider increased with age among the full ADLT population and the ADLT1 + subpopulation $(\mathrm{OR}=0.02,95 \%$ $\mathrm{CI}=1.01-1.02)$ and $(\mathrm{OR}=1.01,95 \% \mathrm{CI}=1.003-1.02)$, respectively; Table 3).

\section{Urgent Care Utilization}

ADLT MRS patients were less likely than non-MRS patients to visit the $\mathrm{UC}(\mathrm{OR}=0.7,95 \% \mathrm{CI}=0.4-1.2)$, although the difference was not significant. In addition, ADLTl+MRS patients were significantly less likely than non-MRS patients to visit the UC $(\mathrm{OR}=0.5,95 \% \mathrm{CI}=0.3-0.9)$. ADLT females were more likely than males to visit the $\mathrm{UC}(\mathrm{OR}=3.5,95 \% \mathrm{CI}=1.6-7.6)$, with a similar difference seen in the ADLTl+ subpopulation $(\mathrm{OR}=3.0,95 \% \mathrm{CI}=1.3-6.7)$. Finally, odds of visiting the UC decreased with age among the full ADLT population and the ADLTl + subpopulation ( $\mathrm{OR}=0.98,95 \% \mathrm{CI}=0.96-0.99)$ and $(\mathrm{OR}=0.97,95 \% \mathrm{CI}=0.96-0.99)$, respectively; Table 3).

\section{Emergency Department Visits: Any Visit}

No significant MRS versus non-MRS or female versus male differences were seen in either the full ADLT or the ADLT1 + subpopulation when evaluating the simple comparison of whether or not any ED visits occurred during the 90 days following the index ED visit (Table 3). However, odds of visiting the ED did decrease with age among the full ADLT population and the 


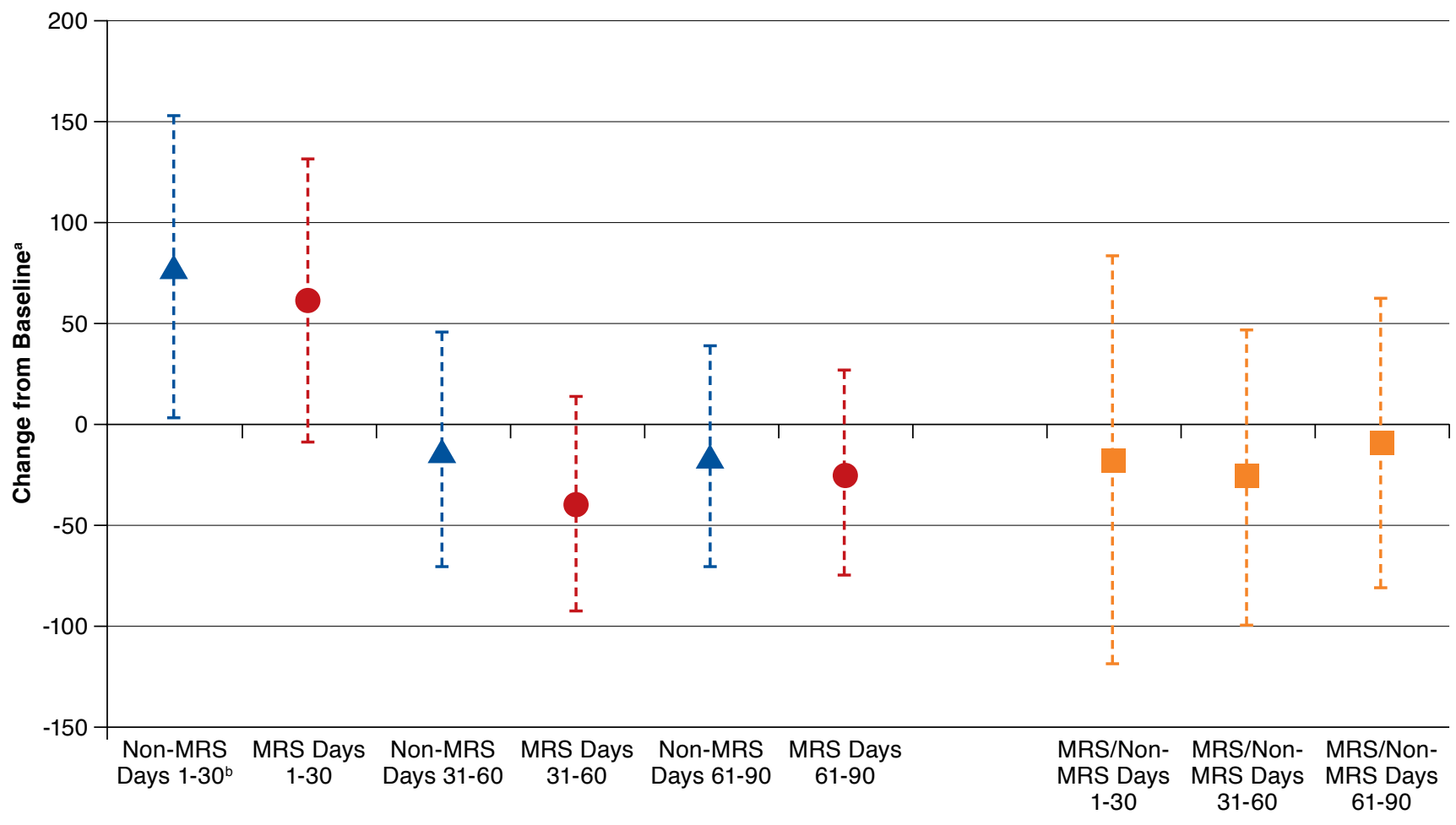

${ }^{a}$ Change from baseline mean of $166.40 \pm 20.95$

${ }^{b}$ Mixed models (repeated measures) linear analysis: $P<0.05$.

$E D=$ emergency department; $M R S=$ medication therapy management and reconciliation service.

ADLT $1+$ subpopulation $(\mathrm{OR}=0.99,95 \% \mathrm{CI}=0.98-0.996$ and $\mathrm{OR}=0.99,95 \% \mathrm{CI}=0.98-0.995$, respectively; Table 3).

\section{Emergency Department Visits: Average Number of Visits}

Figures 1 and 2 present the comparison of the 30-day average number of ED visits (rate per 1,000 persons) from the period before the index ED visit to 1-30, 31-60, and 61-90 days postindex ED visit for both study groups. Each repeated measures linear model was adjusted for patient sex and age (centered at population mean $=48$ ) and weighted by survival time.

Patients in the MRS group had no significant change in the average number of ED visits 1-30, 31-60, and 61-90 days postindex ED visit, compared with the 30-day average from the 90 days preceding the index ED visit in either the full ADLT population (165.9 \pm 120.9 ; Figure 1), or the ADLT1 + subpopulation (189.6 \pm 24.1 ; Figure 2). On the other hand, ADLT nonMRS patients had a significant increase in the average number of ED visits $1-30$ days post-index ED visit $(P=0.042)$, with no other significant differences identified in the ADLT non-MRS patients or the ADLT1+non-MRS patients. Also, while the MRS/non-MRS difference was consistently negative; that is, the change from baseline was lower (rose less or decreased more) for MRS patients, none of these reached the level of statistical significance.

\section{Discussion}

This study evaluated the potential impact of a pharmacist-led patient-centered MRS in collaboration with ED physicians on patient utilization of outpatient health care services.

Based on our analysis, pharmacist provision of a patientcentered MRS in collaboration with ED physicians did not have an overall effect on ED visits. While this finding does not match that of the original pilot study, ${ }^{17}$ it should be understood that the pilot study was conducted prior to passage of the Affordable Care Act, which motivated health system initiation of multiple programmatic changes intended to address the issue of 30-day readmission to the ED. However, the problem facing health systems is not simply to decrease ED utilization. They also seek to improve appropriate patient utilization of available health care services, including PC, UC, and ED. In our analysis, there was an increased odds of PC visits among the MRS group. This is important because a key step in improving care transition 


\section{FIGURE 2 Change from Baseline ${ }^{a}$ : Number of ED Visits (1,000 Persons,} Adjusted for Age and Sex, All Adults with 1+ Medications)

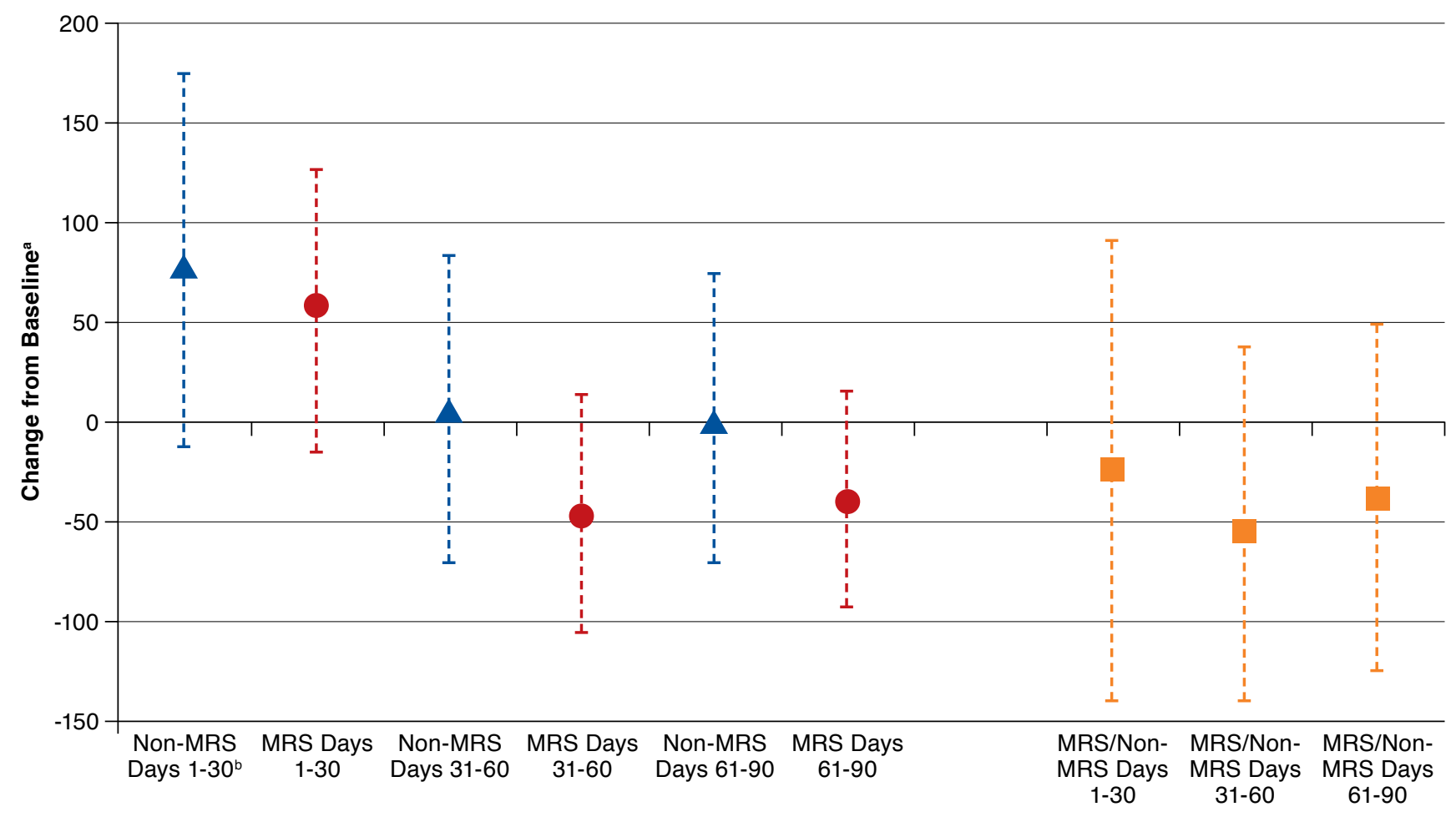

achange from baseline mean of $190.43 \pm 24.17$.

${ }^{b}$ Mixed models (repeated measures) linear analysis: $P<0.05$

$E D=$ emergency department; $M R S=$ medication therapy management and reconciliation service.

is improving outpatient care visits. Among patients taking at least 1 medication, there was also an association between the intervention provided by the pharmacist and decrease in frequency of UC visits. However, this positive effect was not observed in the frequency of ED visits. One possible theory is that there are other social, demographic, or health system factors that may have masked the effect of the intervention provided. Furthermore, at the time of this study, there were policy changes and ongoing quality improvement initiatives in the overall health system that were designed to accomplish the goals of an accountable care organization. The results of this study highlight the difficulty of simply designing a single intervention for reducing ED visits during transition from the ED to home. We hypothesize that as the health care system implements several sets of coordinated interventions or programs, those combined interventions may translate to improved ED utilization. This provides a direction for future research.

It is noteworthy that the increase in PC visits observed in the MRS group has a direct association with involvement of pharmacists with education and provision of letters to both the patient and PC providers. In the MRS intervention group, letters were given to patients with or without a PC provider with an instruction/education to identify a care provider for the management of their disease states. Our analysis did not evaluate the difference between those patients, with and without identified PC providers at the time of the index ED visit. Overall, however, this component of the MRS intervention appears to have contributed successfully to transitioning patients to a PC setting.

Gender and age were the only significant factors identified in the use of PC, UC, and ED visits. As age increases, patients were more likely to use PC and less likely to use either the UC or ED. This is reasonable, since older patients are more likely to have a PC provider when compared with younger patients. ${ }^{12}$ Furthermore, this is consistent with the findings of Doran et al. (2013), who observed an inverse relationship between age and frequency in ED visits. ${ }^{18}$ It is important to note the impact of age in ED visits because interventions designed to improve the frequency of ED visits must consider the impact of this factor as it affects their institution service. Future research will 


\section{Evaluation of the Influence of a Pharmacist-led Patient-Centered Medication Therapy Management and Reconciliation Service in Collaboration with Emergency Department Physicians}

evaluate the impact of insurance and post-index hospitalization on frequency of ED visits.

Implementation of an MRS program in the ED was challenging. First, this was the first time a clinical pharmacist was introduced in the institution's ED to run the MRS program. Logistically, intervention was systematically implemented so that the MRS program would not significantly affect patient length of stay in the ED. The pharmacist was alerted by the research assistant to see each patient before the physician's initial clinical assessment and upon discharge. This was done either by oral communication or via pager. This procedure did not significantly affect length of stay because patients were seen by the pharmacist while waiting for the provider's initial clinical assessment and also while waiting for completion of discharge planning. Furthermore, the pharmacist office was located in the ED, which further enhanced pharmacist access to patients.

Another barrier that we faced prior to implementing the program was identifying the peak hours of admission visits that would be consistent throughout the study period. It tended to vary seasonally (winter vs. summer or spring). Patient enrollment was limited to a 3-month, weekday, and daytime (8:00 Am to 4:00 PM) time period. Therefore, the time period (peak hours) that we chose for clinical pharmacist provision of MRS may not be optimal for other institutions. In addition, despite the randomization of patients to the MRS or non-MRS groups in the ratio of 1:1, we cannot rule out seasonal effect as a contributor to the number or type of patients admitted.

\section{Limitations}

We recognize that this study enrolled patients through a single hospital ED, and the 90-day follow-up was limited to its health system's affiliated facilities (hospitals and clinics). Therefore, a known limitation is that PC, UC, and ED visits outside the health system were not included in the study analyses. This limits the generalizability of our results. Another potential limitation of the study is the unbalanced sample size between the MRS and non-MRS groups. Because the original study randomization was not stratified by age group-child (aged $<18$ years) and adult (aged $>18$ years) - the adult population had slightly more MRS than non-MRS patients. We also observed that the non-MRS group had a higher proportion of patients who reported that they were not taking daily prescribed medications (34.5\%). The cause of this difference remains unclear to study investigators, but this information came from the patient survey, which was completed by the patient or caregiver, and the lack of an MRS review of medications may have impacted responses to this question. Other potential limitations of the study included the small sample size and the short duration of the study. Another factor that may have affected our data is the heterogeneity of the cohort group that was seen (primary diag- nosis). Again, because of the small sample number, we were not able to control for this factor.

\section{Conclusions}

A pharmacist-led patient-centered MRS contributed to increased odds of patients visiting their PC providers. This is a positive first step in transitioning patients toward an appropriate use of PC services, which may contribute toward an improvement in the multifaceted challenge of managing ED visits.

\section{Authors}

ARINZE NKEMDIRIM OKERE, PharmD, MS, BCPS, is Assistant Professor of Pharmacy, Ferris State University College of Pharmacy, Grand Rapids, Michigan; COLLEEN M. RENIER, BS, is Biostatistician, Essentia Health, Essentia Institute of Rural Health, Duluth, Minnesota; and JAMES J. TOMSCHE, PharmD, BCPS, is Director of Pharmacy, Essentia Health, St Mary's Medical Center, Duluth, Minnesota.

AUTHOR CORRESPONDENCE: Arinze Nkemdirim Okere, PharmD, MS, BCPS, Ferris State University College of Pharmacy, 25 Michigan St. NE, Ste. 7000, Grand Rapids, MI 49503.

Tel.: 616.391.0863; Fax: 616.454.2108; E-mail: preseoapc@msn.com.

\section{DISCLOSURES}

Research for this study was funded by Essentia Health Foundation. The authors declare no conflicts of interest.

Study concept and design were primarily contributed by Nkemdirim Okere, along with Renier and Tomsche. Data were collected and analysed by Nkemdirim Okere and Renier. The manuscript was primarily written by Nkemdirim Okere, along with Renier, and was revised equally by Nkemdirim Okere and Renier

\section{ACKNOWLEDGMENTS}

The authors gratefully acknowledge the Pharmacy and Emergency Department of Essentia Health, Duluth, Minnesota, and members of the Essentia Institute of Rural Health clinical research staff.

\section{REFERENCES}

1. Centers for Disease Control and Prevention. National Hospital Ambulatory Medical Care Survey: 2010 Emergency department summary. Available at: http://www.cdc.gov/nchs/data/ahcd/nhamcs_emergency/2010_ed_web_ tables.pdf. Accessed January 19, 2015

2. McCaig LF, Burt CW. National Hospital Ambulatory Medical Care Survey: 2002 emergency department summary. Adv Data. 2004;(340):1-34.

3. Smulowitz PB, O'Malley J, Yang X, Landon BE. Increased use of the emergency department after health care reform in Massachusetts. Ann Emerg Med. 2014;64(2):107-15.

4. Coleman EA, Eilertsen TB, Kramer AM, Magid DJ, Beck A, Conner D. Reducing emergency visits in older adults with chronic illness. A randomized, controlled trial of group visits. Eff Clin Pract. 2001;4(2):49-57. 
5. Coleman EA. Falling through the cracks: challenges and opportunities for improving transitional care for persons with continuous complex care needs. J Am Geriatr Soc. 2003;51(4):549-55.

6. Friedman B, Basu J. The rate and cost of hospital readmissions for preventable conditions. Med Care Res Rev. 2004;61(2):225-40.

7. Rosenthal JM, Miller DB. Providers have failed to work for continuity. Hospitals. 1979;53(10):79-83.

8. Rozich JD, Howard RJ, Justeson JM, Macken PD, Lindsay ME, Resar RK. Standardization as a mechanism to improve safety in health care. Jt Comm J Qual Saf. 2004;30(1):5-14

9. Gleason KM, Groszek JM, Sullivan C, Rooney D, Barnard C, Noskin GA. Reconciliation of discrepancies in medication histories and admission orders of newly hospitalized patients. Am J Health Syst Pharm. 2004;61(16):1689-95.

10. Coleman EA, Smith JD, Raha D, Min SJ. Posthospital medication discrepancies: prevalence and contributing factors. Arch Intern Med. 2005;165(16):1842-47.

11. Vashi AA, Fox JP, Carr BG, et al. Use of hospital-based acute care among patients recently discharged from the hospital. JAMA. 2013;309(4):364-71.

12. Palmer E, Leblanc-Duchin D, Murray J, Atkinson P. Emergency department use: is frequent use associated with a lack of primary care provider? Can Fam Physician. 2014;60(4):e223-29.
13. Werner RM, Canamucio A, Marcus SC, Terwiesch C. Primary care access and emergency room use among older veterans. J Gen Intern Med. 2014;29(Suppl 2):S689-94

14. Grumbach K, Bodenheimer T, Grundy P. The outcomes of implementing patient-centered medical home interventions: a review of the evidence on quality, access and costs from recent prospective evaluation studies, August 2009. Patient-Centered Primary Care Collaborative. Available at: http:// pcmh.ahrq.gov/sites/default/files/attachments/The\%20Outcomes\%20of\%20 Implementing\%20Patient-Centered\%20Medical\%20Home\%20Interventions. pdf. Accessed January 19, 2015.

15. Lowe RA, Localio AR, Schwarz DF, et al. Association between primary care practice characteristics and emergency department use in a Medicaid managed care organization. Med Care. 2005;43(8):792-800.

16. Franco SM, Mitchell CK, Buzon RM. Primary care physician access and gatekeeping: a key to reducing emergency department use. Clin Pediatr (Phila). 1997;36(2):63-68

17. Okere AN, Gessert CE, Renier CM, Swanoski M. Effects of medication reconciliation service provided by student pharmacists in a tertiary care emergency department. Innov Pharm. 2013;4(2):1-9 [article 13]. Available at: http://www.pharmacy.umn.edu/innovations/prod/groups/cop/@pub/@ cop/@innov/documents/article/cop_article_440661.pdf. Accessed January 19, 2015.

18. Doran KM, Raven MC, Rosenheck RA. What drives frequent emergency department use in an integrated health system? National data from the Veterans Health Administration. Ann Emerg Med. 2013; 62(2):151-59. 